\title{
Cloning and Characterization of a Tryptophan- Aspartic Acid Repeat Gene Associated with the Regulation of Anthocyanin Biosynthesis in Oriental Hybrid Lily
}

\author{
Xiaoying Dou \\ Beijing Radiation Center, Beijing 100875, China; and Key Laboratory of Beam Technology of \\ Ministry of Education, Beijing Normal University, Beijing 100875, China \\ Jinrong Bai, Huan Wang, Ying Kong, Lixin Lang, and Fang Bao \\ Beijing Radiation Center, Beijing 100875, China \\ Hongzhong Shang \\ Beijing Radiation Center, Beijing 100875, China; and Key Laboratory of Beam Technology of \\ Ministry of Education, Beijing Normal University, Beijing 100875, China
}

\begin{abstract}
AdDITIONAL INDEX words. flower color, Lilium, LhbHLH2, LhWDR, WD repeat
Abstract. Anthocyanins are major pigments responsible for the color of lily (Lilium sp.) flowers. Anthocyanin synthesis is part of the flavonoid metabolic pathway. Numerous transcription factors, including R2R3-MYBs, basic helix-loop-helix (bHLH), and tryptophan-aspartic acid repeat (also known as WD40 or WD repeat) proteins, known to regulate flavonoid biosynthesis have been identified in various plant species. However, there is limited information available on WD repeat proteins in lilies. In this study, we identified a WD repeat gene in the Oriental hybrid lily 'Sorbonne' (Lilium hybrid WD repeat, $L h W D R$ ). $L h W D R$ contains no introns, and has a 1100-base pair open reading frame, encoding a putative protein of 370 amino acids. LhWDR was found to be localized in the cytoplasm of transgenic Arabidopsis thaliana root cells. Expression patterns of $L h W D R$ in different organs and at different periods of lily tepal growth revealed that the expression levels of this gene are closely associated with anthocyanin accumulation. A yeast two-hybrid assay demonstrated that full-length LhWDR interacts with the $420 \mathrm{~N}$-terminal amino acids of Lilium hybrid bHLH2. Interestingly, overexpression of $L h W D R$ in $A$. thaliana led to an upregulation of the dihydroflavonol 4-reductase gene, which is an important structural gene downstream of the anthocyanin pathway. These results indicate that the WD repeat protein LhWDR might interact with a bHLH transcription factor to regulate anthocyanin biosynthesis.
\end{abstract}

Lilies are popular ornamental plants globally, owing to their range of tepal colors and floral scents. Lilium (Liliaceae) consists of more than 100 species belonging to several sections (Fatihah et al., 2019; Lim et al., 2008). Hybridization within each section is easy, and the hybrids are divided into several groups: longiflorum, trumpet, Oriental, and Asiatic. Oriental hybrid lilies are popular in the cut flower industry because of the aesthetic flower shape, fragrance, and color (Yamagishi and Akagi, 2013). Oriental hybrid lily flowers are mostly white and pink, and anthocyanins are the major pigments that accumulate in the tepals (Wang and Yamagishi, 2019). Anthocyanins are found in a broad range of plant species, in which they contribute to the vivid colors of flowers, including red, pink, purple, and

Received for publication 23 Oct. 2019. Accepted for publication 17 Jan. 2020. Published online 20 February 2020.

This work was supported by the Beijing Academy of Science and Technology Municipal Financial Project (PXM2019_178217_000001), the 12 $2^{\text {th }}$ Five Years Key Programs for Science and Technology Development of China (2013BAD01B0706), and the BJAST Young Researcher Training Plan (201524).

We thank Kezhen Yang for providing Arabidopsis thaliana seeds and transformation vectors and Lei Zhu for her assistance with yeast two-hybrid experiments.

J.B. is the corresponding author. E-mail: bjr301@126.com

This is an open access article distributed under the CC BY-NC-ND license (https://creativecommons.org/licenses/by-nc-nd/4.0/). blue. The colors conferred by anthocyanins to plants are used to attract pollinators and seed-dispersing animals (Winkel-Shirley, 2001) and play important roles in fertility (Grotewold, 2006) and oxidation resistance (Gould et al., 2002).

Anthocyanin biosynthesis is a branch of the flavonoid metabolic pathway, and a range of enzymes are responsible for the synthesis of anthocyanin (Nie et al., 2015). The major enzymes include chalcone synthase (CHS), chalcone isomerase (CHI), dihydroflavonol 4-reductase (DFR), and anthocyanidin synthase (ANS) (Nie et al., 2015). CHS and CHI are early enzymes of anthocyanin biosynthesis, and DFR and ANS are late enzymes. The activity of enzymes involved in anthocyanin biosynthesis is mainly regulated by MYB-bHLH-WD repeat (MBW) ternary complexes composed of R2R3-MYB transcription factors (TFs), a bHLH protein, and WD repeat proteins (Albert et al., 2014; Baudry et al., 2004; Lai et al., 2013; Xu et al., 2015). In plants, R2R3-MYB proteins are among the most abundant transcription factor subfamilies involved in regulating the development of phenotypes, such as plant color (Yamagishi, 2018). The bHLH proteins contain a basic helixloop-helix region, which plays an important role in DNA binding (Nakatsuka et al., 2009). The WD repeat proteins are characterized by WD repeat motif, which is defined as an $\approx 40$-amino acid structure ending with tryptophan-aspartic acid dipeptide (Lloyd et al., 2017). WD repeat proteins generally 
serve as a protein-protein interaction platform for the formation of complexes and as mediators of transient connections between other proteins, thereby stabilizing complexes by binding MYBs or interacting with bHLHs (Lloyd et al., 2017). The mechanisms underlying the regulatory role of the MBW protein complex in anthocyanin biosynthesis have been studied in some plant species (de Vetten et al., 1997; Gonzalez et al., 2008; Morita et al., 2006; Quattrocchio et al., 1999; Schwinn et al., 2006; Spelt et al., 2000; Walker et al., 1999; Zhang et al., 2003). In petunia (Petunia $\times$ hybrida), ANTHOCYANIN 1 (AN1) and ANTHOCYANIN 2 (AN2) encode bHLH and MYB TFs, respectively (Mol et al., 1996), whereas ANTHOCYANIN 11 (AN11) encodes a WD repeat protein (de Vetten et al., 1997). $A N 1, A N 2$, and $A N 11$ form a regulatory network to determine the timing, level, and patterning of anthocyanin accumulation (Albert et al., 2011). In Arabidopsis thaliana, MBW complexes regulate the expression of late anthocyanin synthesis genes to promote anthocyanin production and pigmentation in the seedcoat (Baudry et al., 2004; Gonzalez et al., 2008; Matus et al., 2010).

In lilies, some of the TFs that regulate anthocyanin biosynthesis have been previously described; among which, R2R3MYBs are the most extensively studied. Lilium has several R2R3-MYB TFs with different expression profiles, which contribute to a diverse range of color patterns in the flower tepals (Yamagishi et al., 2018). Lilium MYB12 (LhMYB12) generally regulates the synthesis of anthocyanins in the whole tepals in Asiatic hybrids (Yamagishi et al., 2010, 2012) and Oriental hybrids (Yamagishi, 2011). LhMYB12-Lat regulates the development of splatter-type spots in the Tango Series cultivars of Asiatic hybrid lilies (Yamagishi et al., 2014b), whereas Lilium regale MYB15 (Lr MYB15) is related to the bud-blush pigmentation (Yamagishi, 2016), and LhMYB18 is associated with the big-spot pigmentation in Asiatic hybrid lilies (Yamagishi et al., 2018). Two bHLH-type TFs have been found in lily, and LhbHLH2 is directly related to anthocyanin synthesis (Nakatsuka et al., 2009). It has been confirmed that in Lilium flowers, several R2R3-MYBs control the formation of anthocyanin in conjunction with LhbHLH2 (Nakatsuka et al., 2009; Yamagishi, 2016; Yamagishi et al., 2010, 2014a). So far, the overall molecular mechanisms that underlie tepal pigmentation remain limited. Little is known about the regulation of WD repeat protein in the process of anthocyanin synthesis in lilies.

WD repeat proteins exist in all eukaryotes and participate in a range of biological processes, such as signal transduction, pre-messenger RNA (mRNA) splicing, transcriptional regulation, cytoskeletal assembly, and vesicular trafficking (Neer et al., 1994). WD repeat proteins typically have several conserved regions of 40 to 60 amino acids, the sequences of which start with an N-terminal glycine-histidine (GH) dipeptide and end with a C-terminal WD dipeptide (Kamran et al., 2014; Lai et al., 2012; Neer et al., 1994; Smith et al., 1999). Although WD repeat domains are essential for protein-protein interactions (Yang and Sale, 1998), they lack DNA-binding regions (del March Naval et al., 2016). Recently, the regulation of anthocyanin synthesis by WD repeat protein has been reported in many plants. In petunia, AN11 is a conserved cytoplasmic component of the signal transduction cascade that regulates AN2 function, and thus activates cellular signaling and transcriptional activation (de Vetten et al., 1997). AN11 is capable of intercellular movement in the petal epidermis, which may facilitate anthocyanin pigment pattern formation (Albert et al., 2014). In A. thaliana, TRANSPARENT TESTA GLABRA 1 (TTG1, a WD repeat protein) interacts with R2R3-MYB and bHLH proteins to regulate the production of proanthocyanidin, anthocyanidin, seedcoat mucilage, trichomes, and root hairs (Albert et al., 2014; Feller et al., 2011; Liu et al., 2017). A ttgl mutant has been reported to be defective in anthocyanin function and the production of seedcoats and trichomes (Walker et al., 1999). Overexpression of Fagopyrum tataricum WD40 (FtWD40) in tobacco (Nicotiana tabacum) has been found to induce upregulated expression of the $D F R$ and $A N S$ genes and increase the accumulation of anthocyanins (Yao et al., 2017). However, little is currently known regarding the regulation of WD repeat proteins during the process of anthocyanin synthesis in lilies. To date, based on an analysis of RNAsequencing (RNA-seq) data, Suzuki et al. (2016) found a WD40 gene, which was related to the synthesis of anthocyanin lilies, namely LhWD40a. LhWD40a is a unigene that was identified by blasting the amino acid sequence of TTG1 against the Lollypop sequence database. However, the authors of this study obtained only a part of the coding region of the LhWD40a gene, and did not conduct further research on it. Therefore, more WD repeat genes need to be researched in lilium, which is of great significance for studying the regulation of MBW complex in the anthocyanin synthesis process.

In this study, a novel WD repeat gene, $L h W D R$, was isolated from the Oriental hybrid lily 'Sorbonne'. The relationships between LhWDR and other proteins involved in regulating the anthocyanin biosynthetic pathway were also investigated to determine the function of LhWDR in the regulatory network of anthocyanin biosynthesis.

\section{Materials and Methods}

Plant materials. The Oriental hybrid lily 'Sorbonne' was used for gene cloning and gene expression assays. Bulbs of 'Sorbonne' were purchased from Beijing Plant Horticulture Co. (Beijing, China) and grown in a greenhouse (natural conditions without heating) of the Beijing Radiation Center (Beijing, China). To analyze the gene expression patterns in different organs, we collected the roots, leaves, stems, bulbs, tepals, and anthers of 'Sorbonne'. A. thaliana ecotype Columbia (Col) was grown in soil at $22^{\circ} \mathrm{C}$ under a $16 / 8$-h (light/dark) photoperiod.

IsOLATION OF THE $\boldsymbol{L} \boldsymbol{H} \boldsymbol{W D} \boldsymbol{R}$ GENE. Total RNA was extracted from tepals of 'Sorbonne' at stage 4 (S4) or stage 5 (S5) of growth using an RNeasy Plant Mini Kit (Qiagen, Tokyo, Japan). First-strand DNA was synthesized using a Transcriptor First Strand cDNA Synthesis Kit (Roche, Basel, Switzerland). Degenerate primers were designed based on the sequences of TTG1, AN1 1, Perilla frutescens WD repeat gene (PFWD), Vitis vinifera WD repeat gene ( $V v W D R 1)$, and PALE ALEURONE COLOR1 (PAC1) WD repeat gene from Zea mays. A partial complementary DNA (cDNA) fragment of the $L h W D R$ gene was amplified using the primers LhWDR-C-5' and LhWDR-C-3' (Table 1). The fragment was then inserted into a pEASY-T1 cloning vector using the TA cloning method (TransGen Biotech, Beijing, China), and the recombinant vector was sequenced. The $5^{\prime}$ and $3^{\prime}$ ends of the cDNA sequences were amplified using a SMARTer Rapid Amplification of cDNA ends (RACE) Kit (Clontech, Mountain View, CA). The primers used in this study are listed in Table 1. Full-length cDNA was amplified with rTaq (TaKaRa, Tokyo, Japan) using the primers 
Table 1. Primer names, sequences, and the amplified fragments used for polymerase chain reaction amplification.

\begin{tabular}{|c|c|c|}
\hline Primer names & Primer sequences $\left(5^{\prime}-3^{\prime}\right)$ & Amplified fragments \\
\hline LhWDR-C-5' & GTCACMTAYGATCMYC & A partial complementary DNA (cDNA) of Lilium hybrid \\
\hline LhWDR-C-3' & GACATBGGATCAATCCC & tryptophan-aspartic acid repeat (LhWDR) gene \\
\hline LhWDR-GSP3-1 & CACGCAATTGCATTAACCCCAGCCT & The $3^{\prime}$ end of $L h W D R$ cDNA \\
\hline LhWDR-GSP5-1 & AGTTCTGCGCTCCCCTCACCTCCTT & The $5^{\prime}$ end of $L h W D R$ cDNA \\
\hline LhWDR-GSP5-2 & GACTTCCTCCGTATCTGGCGCATCTCC & \\
\hline LhWDR-F & TCTAGAGAAATGGGCGCCAGCGCTGCC & The full-length cDNA of $L h W D R$ gene \\
\hline LhActin-RT1 & CCTAACCGATTCTCTGATGAAGAT & A fragment of Lilium Actin gene \\
\hline LhActin-RT2 & TGTATACCAGTAGCTTCCATTCCA & \\
\hline LhWDR-RT1 & GCAAGGACAAGAAGTACCGC & A fragment of $L h W D R$ \\
\hline LhWDR-RT2 & GGGAATGAACATGGATTTGG & \\
\hline LhWDR-CDS-F & TCTAGAGAAATGGGCGCCAGCGCTGCC & The full-length coding sequence (CDS) of $L h W D R$ \\
\hline LhWDR-CDS-R & GGTACCAACCCGGAGTATCTGCAGCTT & \\
\hline LhbHLH2-Y-F421 & TCTGAAGGGACTTCACAAAGGATG & A fragment encoding the C-terminal 260 amino acids of LhbHLH2 \\
\hline LhbHLH2-Y-R1 & CTGAGAGAAGATATAATGAATTGC & for $\mathrm{Y} 2 \mathrm{H}$ \\
\hline LhbHLH2-RT1 & AGATCTGGGACTGGTACAGCAT & A fragment of $L h b H L H 2$ gene \\
\hline LhbHLH2-RT2 & GACCCTTGAAGGTTGATAGTCG & \\
\hline LhMYB12-RT1 & GAAGACAACCTCCTCCGCAA & A fragment of Lilium $M Y B 12(L h M Y B 12)$ gene \\
\hline LhMYB12-RT2 & TCCTCCTCATCCTCGCTGAA & \\
\hline LhCHS-RT1 & TCAAGAGGATGTGCGACAAG & A fragment of Lilium chalcone synthase (LhCHS) gene \\
\hline LhCHS-RT2 & GGAGCTTGGTGAGCTGGTAG & \\
\hline LhCHI-RT1 & TCGAAGGATGGCTTGATACC & A fragment of Lilium chalcone isomerase ( $\mathrm{LhCHI}$ ) gene \\
\hline LhCHI-RT2 & CCTTGAGAAGCTCGGAAATG & \\
\hline LhDFR-RT1 & GAACGGCTAACCATTTGGAA & A fragment of Lilium dihydroflavonol 4-reductase (LhDFR) gene \\
\hline DFR-RT1 & ATGCCGCCTAGCCTTATCAC & A fragment of $A$. thaliana $D F R$ gene \\
\hline DFR-RT2 & CCCATGTCCGTCAGCTTCTT & \\
\hline ANS-RT1 & AAGGCTCTCTCTGTCGGTCT & A fragment of $A$. thaliana $A N S$ gene \\
\hline ANS-RT2 & GTGACCCATTTGCCCTCGTA & \\
\hline AtPAP1-RT1 & AGCATCAGTCGACAGGCAAA & A fragment of $A$. thaliana PURPLE ACID PHOSPHATASE 1 gene \\
\hline AtPAP1-RT2 & CCTCCACCTCCAGCAACAAT & \\
\hline GL3-RT1 & ACAGCTTCTCCGAGCAGAAC & A fragment of $A$. thaliana $G L A B R A 3$ gene \\
\hline GL3-RT2 & CGCCCCTTCAACAAACGTTT & \\
\hline $18 \mathrm{~S}$ rRNA & CGGCTACCACATCCAAGGAA & A fragment of $18 \mathrm{~S}$ ribosomal RNA \\
\hline 18S rRNA & TGTCACTACCTCCCCGTGTCA & \\
\hline
\end{tabular}

LhWDR-F and LhWDR-R (Table 1). The DNA fragment thus obtained was subsequently sequenced. Genomic DNA was isolated from the young leaves of 'Sorbonne' using the DNA Plant System Kit (Tiangen, Beijing, China). Sequencing and primer synthesis were performed by the Majorbio Biology Technology Co. (Beijing, China).

Sequence analysis. Multiple WD repeat sequences were aligned using DNAMAN 8 (Lynnon Biosoft, San Ramon, CA). A phylogenetic tree was constructed using the neighbor-joining method (Saitou and Nei, 1987) and was visualized using MEGA version 6. Tree nodes were evaluated for 1000 repli- cates via bootstrap analysis. Publicly available sequences were obtained from GenBank via the National Center for Biotechnology Information (Bethesda, MD). The amino acid sequence was analyzed using the MyHits website of the Swiss Institute of Bioinformatics (University of Lausanne, Lausanne, Switzerland).

DETERmination OF TOTAL ANTHOCYANin CONTENT. The tepals of 'Sorbonne' flowers were ground in liquid nitrogen, and the total anthocyanin content was determined according to the method of Rabino and Mancinelli (1986). We added $5 \mathrm{~mL}$ of acidic methanol solution $(1 \% \mathrm{HCl}, \mathrm{v} / \mathrm{v})$ per gram of tepals 
(A)
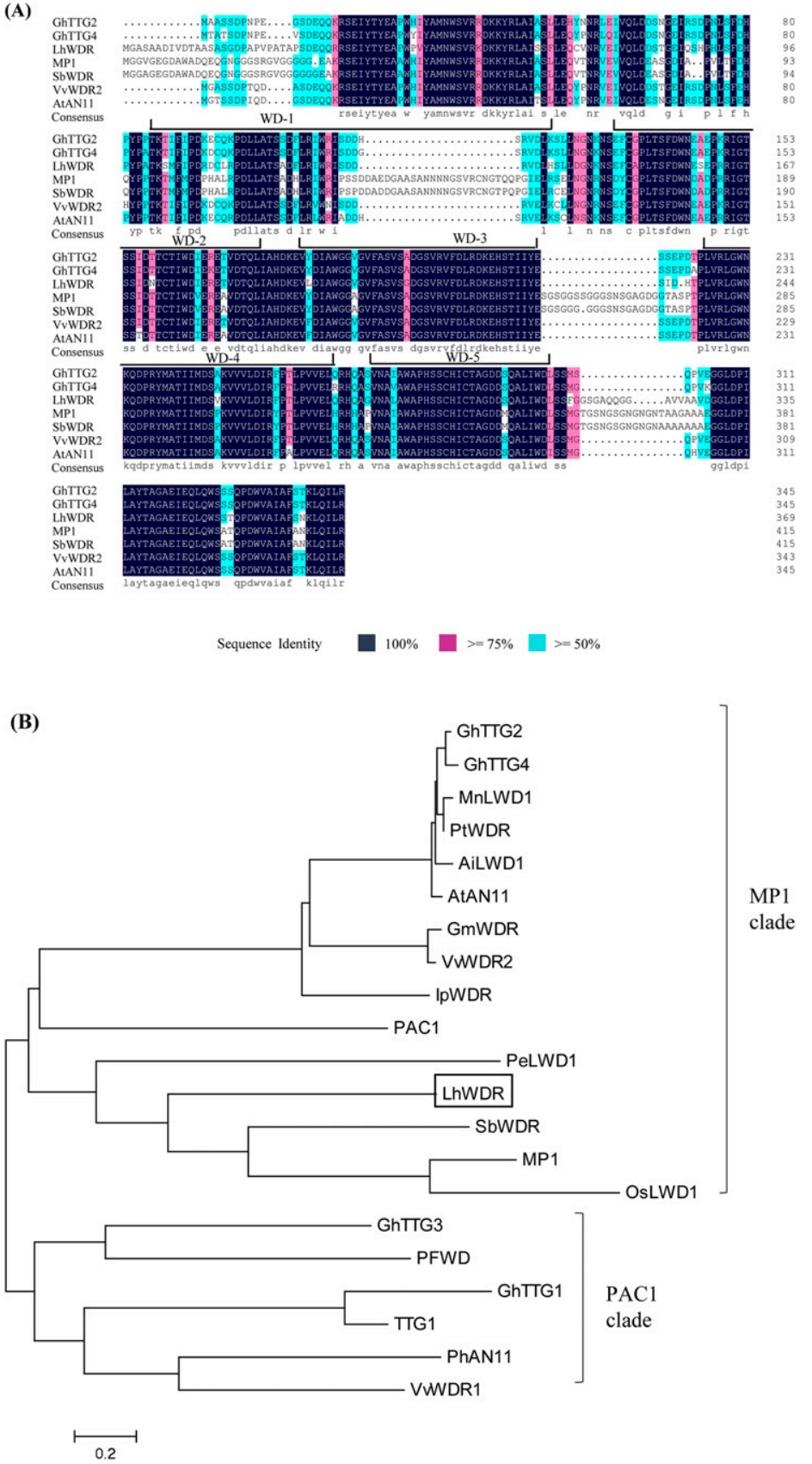

Fig. 1. Protein sequence alignment and phylogenetic tree of Lilium hybrid WD repeat (LhWDR) and selected tryptophan-aspartic acid (WD) repeat domains. (A) The deduced protein sequence of LhWDR was compared with the sequences of Gossypium hirsutum TRANSPARENT TESTA GLABRA 2 (GhTTG2), G. hirsutum TRANSPARENT TESTA GLABRA 4 (GhTTG4), Zea mays MP1, Sorghum bicolor WD repeat (SbWDR), Vitis vinifera WD repeat 2 (VvWDR2), and Arabidopsis thaliana ANTHOCYANIN 11 (AtAN11). Predicted WD repeats are labeled (WD-1 to WD-5). (B) The tree was constructed using the neighbor-joining method and bootstrapped with 1000 replicates. The scale bar indicates the branch length that corresponds to the number of substitutions per amino acid position. The names and GenBank accession numbers are as follows: GhTTG2, AAM95644; GhTTG4, AAM95646; Morus notabilis WD repeat (MnLWD1), XP_010090944; Populus trichocarpa WD repeat (PtWDR), XP_002301564; Arachis ipaensis WD repeat 1 (AiLWD1), XP_016162111; AtAN11, AAC18912; Glycine max WD repeat (GmWDR), XP_003553993; VvWDR2, XP 010660592; Ipomoea batatas WD repeat (IpWDR), AHM25607; Z. mays PALE ALEURONE COLOR1 (PAC1), AAM76742; Populus euphratica WD repeat 1 (PeLWD1), XP_011003601; LhWDR, KY706354; SbWDR, XP 002453914; MP1, AY339884; Oryza sativa Japonica group WD repeat 1 (OsLWD1), XP_015627230; G. hirsutum TRANSPARENT TESTA GLABRA 3 (GhTTG3), AAM95645; Perilla frutescens WD repeat gene (PFWD), BAB58883; G. hirsutum TRANSPARENT TESTA GLABRA 1 (GhTTG1), AAM95641; $A$. thaliana TRANSPARENT TESTA GLABRA 1 (TTG1), CAB45372; Petunia $\times$ hybrida ANTHOCYANIN 11(PhAN11), AAC18914; and $V$. vinifera WD repeat 1 (VvWDR1), ABF66625. The proteins in parentheses indicated that they were clustered in PALE ALEURONE COLOR1 or MP1 clade. material and shook at $4{ }^{\circ} \mathrm{C}$ for $48 \mathrm{~h}$. Absorbances of the filtered extracts were subsequently measured at wavelengths of 530 and $657 \mathrm{~nm}$. The anthocyanin contents in the preparations were determined using the formula: $\left(\mathrm{A}_{530}-0.25 \mathrm{~A}_{657}\right) / \mathrm{M}$, in which $\mathrm{A}_{530}$, and $\mathrm{A}_{657}$ are the absorptions at 530 and $657 \mathrm{~nm}$, respectively, and $\mathrm{M}$ is the weight of tepals (grams). For each sample, we performed determinations for three replicates.

Gene eXPRession anALYSIS. The roots, stems, bulbs, leaves, anthers, and tepals of 'Sorbonne' were harvested immediately after flowering (S5). Total RNA was obtained as previously described for isolating the $L h W D R$ gene. Approximately 2 $\mu \mathrm{g}$ of total RNA was used to synthesize first-strand cDNA using a Transcriptor First Strand cDNA Synthesis Kit and an anchored-oligo $(\mathrm{dT})_{18}$ primer. The reaction product thus obtained was then directly used as a template for reverse-transcription polymerase chain reaction (RTPCR) and quantitative real-time PCR amplification.

RT-PCR was performed using 20 $\mu \mathrm{L}$ reaction solution containing 1 $\mu \mathrm{L}$ of first-strand cDNA and $2 \times$ TransTaq-T PCR SuperMix (TransGen Biotech). Lilium Actin gene (LhActin) cDNA was used as an internal control to normalize the amounts of cDNA. The primers LhActin-RT1 and LhActin-RT2 (Table 1) were used to amplify the LhActin cDNA fragment. The primers LhWDR-RT1 and LhWDRRT2 (Table 1) were used to amplify the $L h W D R$ cDNA fragment. Assessments were carried out as described by Yang et al. (2009). The expression levels of $L h W D R$, LhbHLH2, LhMYB 12, LhCHS, LhCHI, LhDFR, and LhANS were determined using $20-\mu \mathrm{L}$ reaction solutions containing $2 \mu \mathrm{L}$ of $10 \times$ diluted first-strand cDNA and a LightCycler 480 SYBR Green I master mix (Roche, Basel, Switzerland). PCR amplification was performed in a real-time PCR instrument (480, Roche) using the following program: $95{ }^{\circ} \mathrm{C}$ for 10 mins, followed by 40 cycles of $95^{\circ} \mathrm{C}$ for $30 \mathrm{~s}$ and $60{ }^{\circ} \mathrm{C}$ for $1 \mathrm{~min}$.

LhActin levels were quantified as an internal control for lily gene 


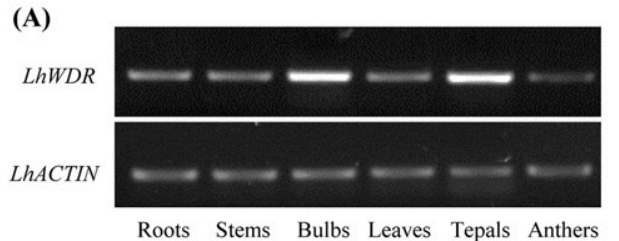

(B)

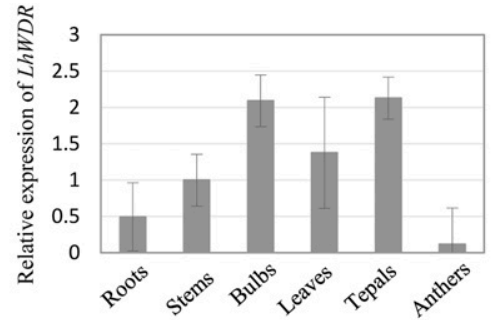

(C)
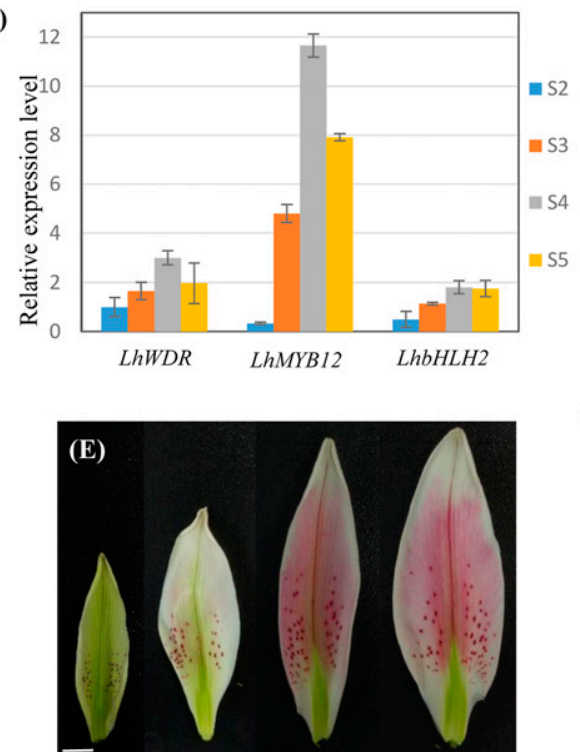

S2

S5
(D)

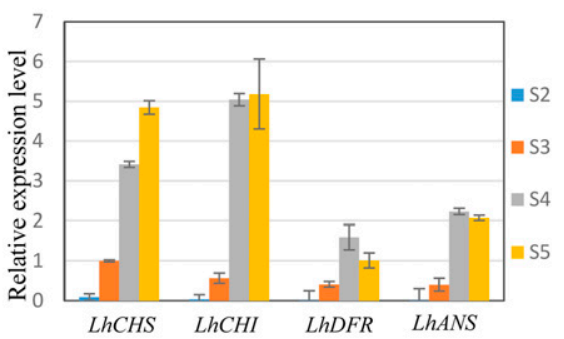

(F)

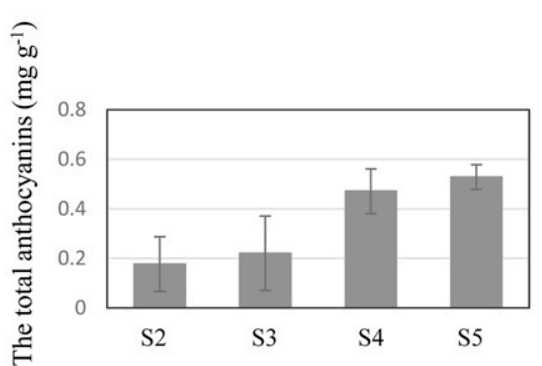

Fig. 2. Expression patterns of Lilium hybrid WD repeat (LhWDR). (A) Reverse-transcription polymerase chain reaction (RT-PCR) assay to determine $L h W D R$ mRNA levels in roots, stems, bulbs, leaves, tepals, and anthers. (B) Quantitative real-time PCR assays to determine the relative expression of $L h W D R$ in roots, stems, bulbs, leaves, tepals, and anthers. (C and D) Quantitative real-time PCR assays to determine the relative expression levels of $L h C H S, L h C H I, L h D F R, L h A N S, L h W D R$, LhMYB12, and LhbHLH2 in stage 2 (S2) to stage 5 (S5) tepals. (E) The process of 'Sorbonne' inner tepal coloring. (F) Quantification of total anthocyanins in 'Sorbonne' inner tepals at S2 to S5 of development. Each value represents the mean of three replicates, and error bars indicate standard deviations $( \pm \mathrm{SD})$; white bar $=1 \mathrm{~cm}$.

expression to normalize the RNA levels. The primers used to amplify the genes LhWDR, LhbHLH2, LhMYB12, LhCHS, $L h C H I, L h D F R$, and $L h A N S$ are listed in Table 1 . The relative mRNA levels of the reaction products were calculated using the comparative $C$ t method (Liu et al., 2007). All PCRs were performed in triplicate and repeated twice.

The gene names and accession numbers are as follows: LhWDR (KY706354), LhActin (AB438963) LhbHLH2 (BAE20058), LhMYB12 (BAJ05398), LhCHS (HQ161731), LhCHI (KJ784468), LhDFR (BAM28975), and LhANS (BAM28976).

DETERMination OF PROTEIN SUBCELlULAR LOCALIZATION. The $L h W D R$ open reading frame (ORF) lacking stop codon was amplified with rTaq using the primers LhWDR-CDS-F and LhWDR-CDS-R (Table 1). After verification by sequencing, the $L h W D R$ coding sequence (CDS) fragment was cloned in front of the GREEN FLUORESCENT PROTEIN (GFP) sequence in a modified pCAMBIA1300 vector containing the Cauliflower mosaic virus $35 \mathrm{~S}$ promoter and a GFP-coding region, resulting in the $35 \mathrm{~S}: L h W D R-G F P$-NOS construct. This construct was initially transformed into Agrobacterium tumefaciens (strain GV3101) and then into $A$. thaliana wild-type Col plants via Agrobacterium-mediated flower bud transformation

(Bechtold and Pelletier, 1998). The transformed plants were selected using $20 \mathrm{mg} \cdot \mathrm{L}^{-1}$ hygromycin. The localization of the LhWDR-GFP fusion protein in the roots of transgenic plants was observed using a confocal microscope LSM510 (Carl Zeiss, Oberkochen, Germany).

ECTOPIC EXPRESSION OF LHWDR IN $\boldsymbol{A}$. THALIANA. The 35S:LhWDRGFP-NOS transgenic plants were used to analyze the effects of related genes in $A$. thaliana with wild-type plants being used as a control. We selected four $\mathrm{T}_{2}$ lines homozygous for the insertion with strong fluorescence for detection, which were named OE1 to OE4 with wild-type plants being used as a control. The method used for the determination of A. thaliana CHS (AtCHS), A. thaliana (AtCHI), DFR, ANS, A. thaliana PURPLE ACID PHOSPHATASE 1 (AtPAP1), and GLA$B R A 3$ (GL3) gene expression levels was as described previously. The $18 \mathrm{~S}$ ribosomal RNA (18S rRNA) level was used as internal control to normalize the RNA levels. The primers used to amplify the $A$. thaliana genes are listed in Table 1. The gene names and accession numbers are as follows: AtCHS (At5g13930), AtCHI (At3g55120), DFR (At5g42800), $A N S$ (At4g22880), AtPAP 1 (At1g13750), and GL3 (At5g41315). Three independent plants of each homozygous $T_{2}$ insertion line were selected for biological replication.

Yeast TWO-HYBRID ASSAYS. The Gal4 vector system was used for yeast two-hybrid ( $\mathrm{Y} 2 \mathrm{H})$ assays (Clontech, Mountain View, CA). The CDS of $L h W D R$ was cloned into the pGADT7 vector. The full-length CDS and a CDS fragment of $L h b H L H$ were cloned into the pGBKT7 vector. These constructs were cotransformed into the yeast strain Y187 using the PEG/LiAC method, as described by Causier and Davies (2002). The transformed Y187 cells were adjusted to an OD600 of $0.3-0.4$ and then cultured on synthetic medium plates lacking tryptophan and leucine acid (SD/-Trp/-Leu) and synthetic medium plates lacking tryptophan, leucine, histidine, and adenine acid (SD/-Trp/-Leu/-His/-Ade) plates for 2 to $6 \mathrm{~d}$ at $30^{\circ} \mathrm{C}$. The primers used in this experiment are shown in Table 1.

\section{Results}

Cloning of the LHWDR gene from the tepals OF ORIENTAL HYBRID LILY. Our primary aim was to isolate a WD repeat gene that may be involved in anthocyanin biosynthesis in Lilium. Using homology cloning and RACE technology, fulllength cDNA of the $L h W D R$ gene was isolated from the tepals of Oriental hybrid lily 'Sorbonne', including 15 base pairs (bp) 


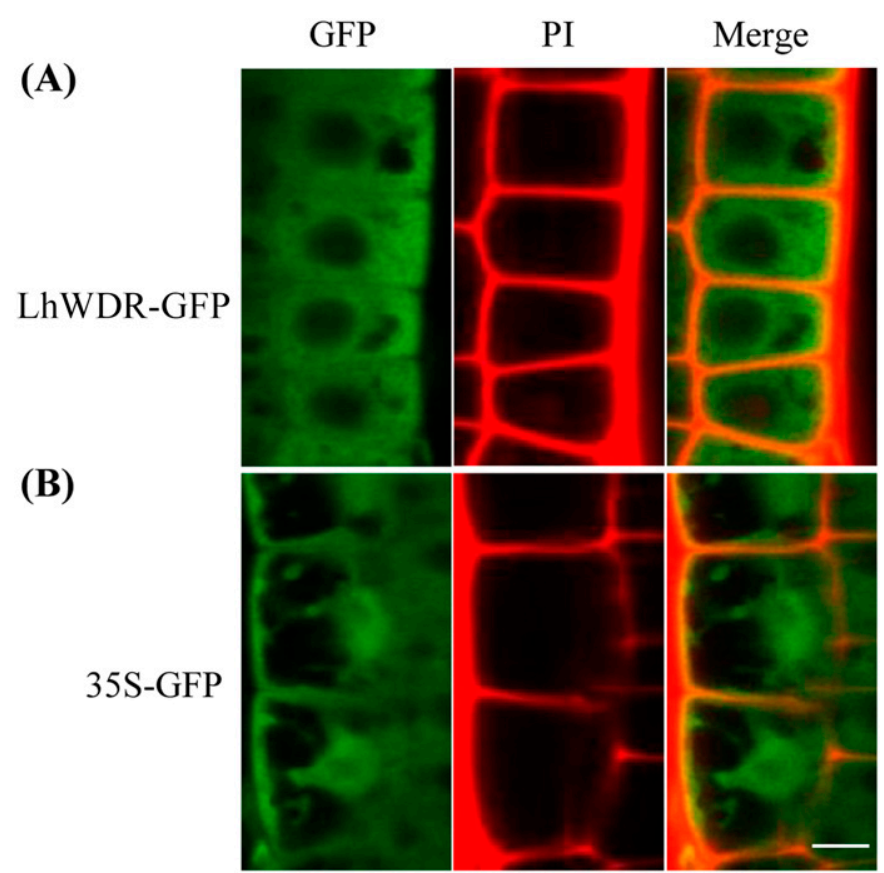

Fig. 3. Subcellular localization of Lilium hybrid WD repeat-green fluorescent protein (LhWDR-GFP) in Arabidopsis thaliana root cells. (A) The GFP signals of LhWDR-GFP were located in the cytoplasm of $A$. thaliana root cells. (B) Signals of 35S-GFP (positive control). Propidium iodide (PI) was used to stain the cell profile and the signal was merged with the GFP signal in the right hand column. Scale bar $=1 \mu \mathrm{m}$.

of the $5^{\prime}$ untranslated region (UTR), $1110 \mathrm{bp}$ of CDS, and 244 bp of 3' UTR. Comparisons of the genomic DNA and cDNA sequences indicated that $L h W D R$ lacks introns, as has previously been observed for TTG1 and $A N 11$. The sequence of LhWDR has been deposited in GenBank under the accession number KY706354.

Sequence and Phylogenetic analyses. Sequence analysis revealed that the full-length cDNA of $L h W D R$ consists of a 1110-bp ORF that encodes a predicted protein of 370 amino acids (Fig. 1A). The results of a basic local alignment search tool revealed that LhWDR contains five WD repeat sequences, and the deduced amino acid sequence indicated sequence similarity with other members of the WD protein family. The amino acid sequence was highly conserved with respect to WD repeats 2-5 compared with those of WD repeat proteins from A. thaliana, Z. mays, $V$. vinifera, and Gossypium hirsutum (Fig. 1A). Most of the homology was observed within the WD repeat motifs. The core length of the WD repeat motifs was $\approx 40$ amino acids, and these motifs were characterized by $\mathrm{GH}$ and WD doublet residues. Multiple sequence alignment of the deduced amino acid sequences revealed that LhWDR shared $83 \%$ amino acid identity with the $V$. vinifera WD repeat 2 (VvWDR2) protein, 81\% with $A$. thaliana AN11 (AtAN11), $80 \%$ with $G$. hirsutum TRANSPARENT TESTA GLABRA 4 (GhTTG4), 65\% with Z. mays MP1 and Sorghum bicolor WD repeat (SbWDR) (Fig. 1A). WD repeat proteins associated with plant flavonoids are classified in the 4WD-repeat subfamily, which is divided into the PAC1 and MP1 clades (Carey et al., 2004). The phylogenetic tree we constructed using LhWDR and homologous proteins from fourteen other species (Fig. 1B) revealed that LhWDR clustered in the MP1 clade, which included VvWDR2, MP1, and AtAN11.
Expression PATTERNS OF LHWDR AND ANTHOCYANINRELATED GENES. The expression pattern of the $L h W D R$ gene was observed during plant development and inflorescence. RTPCR and quantitative real-time PCR were performed to evaluate the expression levels of $L h W D R$ in different plant tissues. The results revealed that $L h W D R$ is expressed in all the tissues we examined (Fig. 2A), which is similar to the expression patterns of the constitutively expressed genes $A N 11$ and PFWD (de Vetten et al., 1997; Sompornpailin et al., 2002). However, the expression levels of $L h W D R$ varied in different tissues, with the pink tepals and light-pink bulbs exhibiting higher transcript levels than other tissues [roots, leaves, stems, and anthers (Fig. $2 \mathrm{~A}$ and B)], suggesting that the expression of $L h W D R$ may be associated with pigmentation.

The transcription profiles of the genes during flower development from S2 to S5 were also investigated using quantitative real-time PCR. Purple spots began to appear on the tepals of 'Sorbonne' from S2 and the tepals became pale pink at S3, and had turned dark pink by S5 (Fig. 2E). During the period of development from S2 to S5, we detected a gradual increase in the total content of anthocyanins (Fig. 2F). Quantitative realtime PCR results showed that there was an accumulation of $L h W D R$ transcripts from S2 to S5, with transcription peaking at S4 (Fig. 2C). Furthermore, the transcription profiles of LhMYB 12 and LhbHLH2 were found to be similar to the transcription profile of $L h W D R$, in that transcription peaked at S4. However, the increase in LhMYB12 transcript levels was higher than that in the transcript levels of $L h W D R$ and LhbHLH2. Subsequent analysis of four key structural genes from lilies predicted to be involved in flavonoid and anthocyanin biosynthesis, namely, LhCHS, LhCHI, LhDFR, and $L h A N S$, revealed that the transcription of these genes was low or undetectable at S2 and highest at S4 or S5. Moreover, expression of the $L h D F R$ and $L h A N S$ genes was observed to be similar to that of $L h M Y B 12, L h b H L H 2$, and $L h W D R$ (Fig. 2D). The similarity of these expression patterns accordingly indicated that $L h W D R$ may be associated with the anthocyanin metabolic pathway.

Subcellular localization OF THE LhWDR PRotein. To investigate the subcellular localization of LhWDR, the p35S:LhWDR-GFP construct was transformed into wild-type A. thaliana and a total of 47 transgenic plants were subsequently obtained. Observation of the root tips of $T_{1}$ transgenic plants revealed that 31 plants showed green fluorescence, and PCR analysis indicated that all of these plants had the transgene insertion. The GFP signal was observed in transformed root cells by comparison with cells stained with propidium iodide. The LhWDR-GFP fusion proteins were observed under fluorescent light as green signals localized in the cytoplasm (Fig. 3A), whereas the signals obtained with the control vector $35 \mathrm{~S}: G F P$ were distributed throughout the cells (Fig. 3B). These results are consistent with those obtained for $A N 11$ and PFWD, and the proteins encoded by these genes were also detected in the cytoplasm (de Vetten et al., 1997; Sompornpailin et al., 2002).

ECTOPIC EXPRESSION OF LHWDR PROMOTES DFR GENE EXPRESSION IN $\boldsymbol{A}$. THALIANA. Observations of $\mathrm{T}_{1}$ plants harboring the p35S:LhWDR-GFP construct throughout the growth cycle revealed no significant phenotypic changes. To characterize the function of $L h W D R$ in anthocyanin biosynthesis, four $\mathrm{T}_{1}$ plants with strong GFP fluorescence (OE1-OE4) were selected to obtain homozygous $\mathrm{T}_{2}$ generation plants by selfing. The homozygous transgenic insertion was selected for gene detection 
(A)

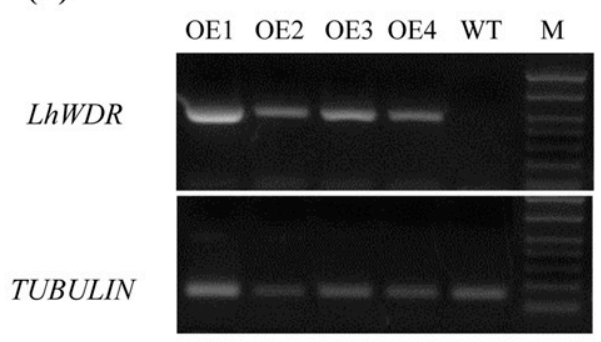

(B)

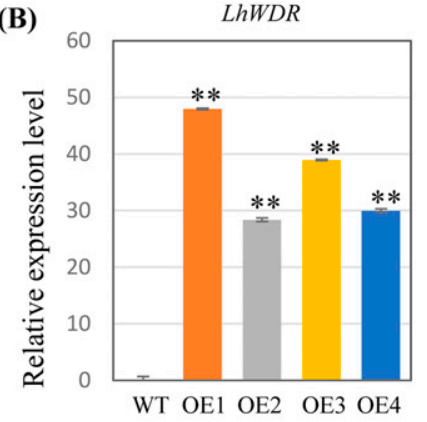

(C)

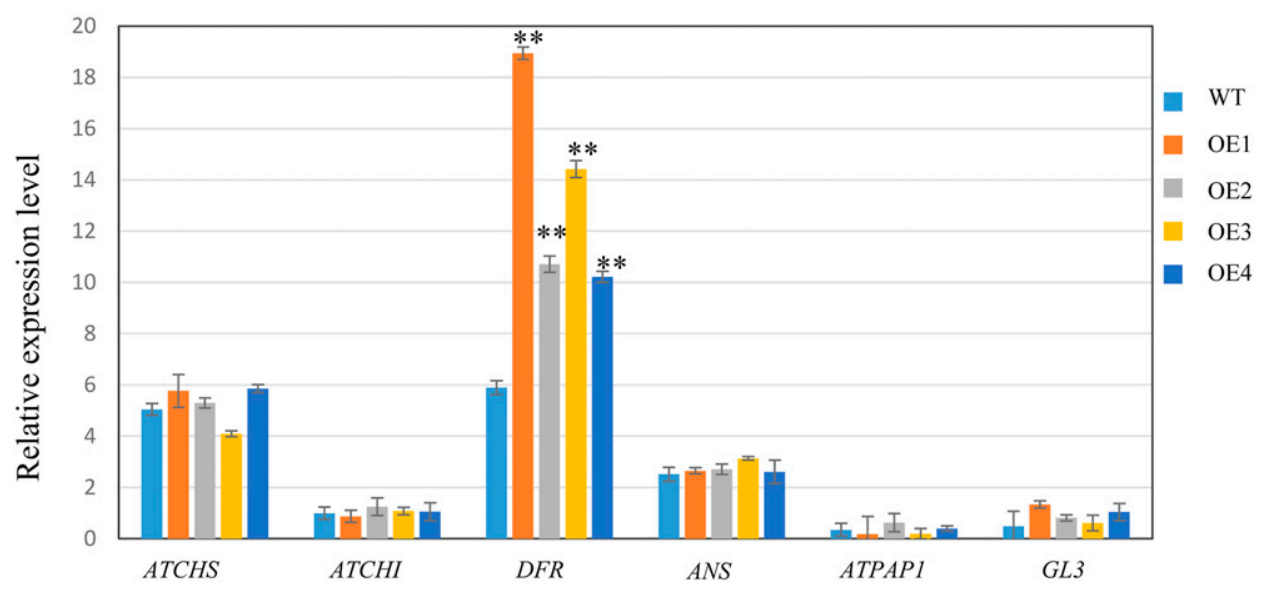

Fig. 4. Expression levels of related genes in Arabidopsis thaliana overexpressing Lilium hybrid WD repeat (LhWDR). Reverse-transcription polymerase chain reaction (RT-PCR) (A) and quantitative real-time PCR (B) assays to determine $L h W D R$ mRNA levels in wild-type plant (WT) and $L h W D R$-overexpressing plants (OE1OE4). (C) Expression levels of AtCHS, AtCHI, DFR, ANS, AtPAP1, and GL3 in WT and OE1-OE4 plants. **Significant difference at the $1 \%$ level ( $t$-test).

and we found that the $L h W D R$ gene was overexpressed in the $\mathrm{T}_{2}$ OE1-OE4 lines (Fig. 4A and B). Quantitative real-time PCR analysis of the expression levels of the anthocyanin biosynthesis genes $A T C H S, A T C H I, D F R$, and $A N S$; the regulatory MYB TF gene ATPAPI; and the bHLH gene GL3 revealed that the average expression level of $D F R$ in the transgenic plants was twice that in the control plants (Fig. 4C), whereas no clear changes were observed in the expression of the other genes (Fig. 4C). Our findings indicated that overexpression of the $L h W D R$ gene upregulates $D F R$, a key gene in late anthocyanin synthesis in $A$. thaliana, and we thus speculate that $L h W D R$ may participate in the anthocyanin synthesis pathway by regulating the transcription of $D F R$.

THE LHWDR PROTEIN INTERACTS WITH LHBHLH2 IN YEAST. In A. thaliana, TTG1 has been shown to interact with the bHLH TFs GL3 and ENHANCER OF GLABRA3 (EGL3) to regulate the synthesis of procyanidins and the formation of epidermal hairs (Walker et al., 1999; Zhang et al., 2003). To date, two bHLH TFs have been studied in Asiatic hybrid lilies ( $L h b H L H 1$ and $L h b H L H 2$ ); however, only $L h b H L H 2$ has been reported to regulate anthocyanin metabolism, via regulating the expression of the downstream gene LhDFR (Nakatsuka et al., 2009). To examine the relationship between LhWDR and LhbHLH2, we used a Y2H system. LhbHLH2 is a self-activating protein (Fig. 5) owing to the bHLH structure, which is 420 to 600 amino acids long. Therefore, the sequence of LhbHLH2 was divided into two fragments when constructing the vectors. The N-terminal 420 amino acids were cloned into pGBKT 7 to construct the LhbHLH2-420-BD vector, whereas the C-terminal 260 amino acids were cloned into pGBKT7 to construct the LhbHLH2-260-BD vector. The results showed that the fragment comprising the N-terminal 420 amino acids did not exhibit selfactivation, whereas the C-terminal 260 amino acids fragment was selfactivated (Fig. 5). The full-length LhWDR was cloned into pGADT7 to construct the LhWDR-AD vector and the $\mathrm{Y} 2 \mathrm{H}$ assay revealed that this full-length LhWDR could interact with the N-terminal 420 amino acids of the LhbHLH2 protein (Fig. 5).

\section{Discussion}

WDR ANTHOCYANIN REGULATORY PROTEINS ARE HIGHLY CONSERVED. WD repeat motifs are typically 40 to 60 amino acids in length, and the 40- to 60-residue sequence generally contains a GH dipeptide in the $\mathrm{N}$-terminal region and a WD dipeptide in the C-terminal region (Kamran et al., 2014; Smith et al., 1999). Studies have reported that the motif generally consists of 4 to 10 tandemly repeated units that form a beta-propeller structure (van Nocker and Ludwig, 2003). In the present study, we cloned an anthocyanin biosynthesis regulatory gene $(L h W D R)$ belonging to the WD repeat gene family, which is characterized by five WD repeat sequences. In each WD repeat motif of the LhWDR protein, the core length between the GH and WD sequences is $\approx 40$ amino acids. We found that WD repeats 2 to 5 have $>80 \%$ identity with the corresponding repeats in WD regions of homologous proteins. Plant flavonoid-related WD repeat proteins have been grouped into two clades, namely, the PAC1 and MP1 clades (Matus et al., 2010), and the phylogenetic tree we constructed revealed that LhWDR clustered in the MP1 clade. It is predicted that the members of both PAC1 and MP1 clades function in DNA and protein binding, and based on sequence alignment, it has been found that the two clades differ with respect to the number and position of WD repeat domains. Previous analysis has revealed that MP1 clade sequences lack nuclear localization signals (Yao et al., 2017), and we have found that neither PAC1 nor MP1 exhibit typical nuclear localization signals. Both AN11 and $P F W D$ belong to the PAC1 clade, and the proteins encoded by these genes have been detected in the cytoplasm (de Vetten et al., 1997; Sompornpailin et al., 2002). Based on subcellular localization experiments, we confirmed that LhWDR is localized in the cytoplasm rather than in the nucleus.

To date, there have been numerous studies that have focused on the genes of WD repeat proteins in the PAC1 cluster, among 

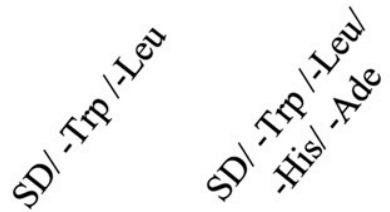

$\mathrm{AD} / \mathrm{BD}$
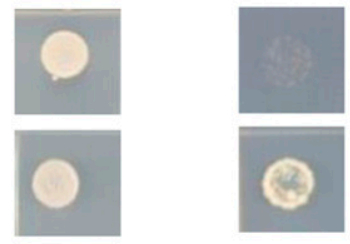

$\mathrm{AD} /$

LhbHLH2

$\mathrm{AD} /$

LhbHLH2-420

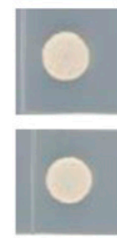

LhbHLH2-260

LhWDR / BD

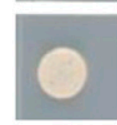

LhWDR /

LhbHLH2-420
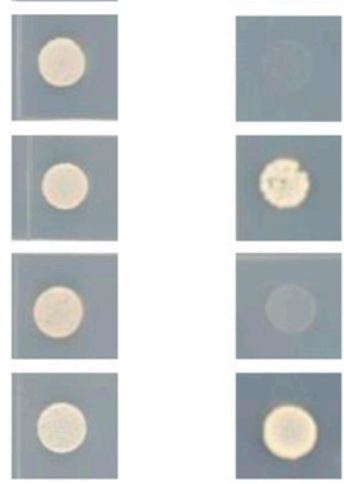

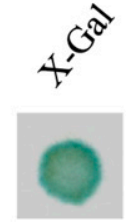

Fig. 5. Yeast two-hybrid analysis of protein-protein interactions. The cotransformed yeast cells were grown on synthetic medium plates lacking tryptophan and leucine acid (SD/-Trp/-Leu) at $30{ }^{\circ} \mathrm{C}$ for $2 \mathrm{~d}$ and synthetic medium plates lacking tryptophan, leucine, histidine, and adenine acid (SD/-Trp/-Leu/-His/Ade) at $30^{\circ} \mathrm{C}$ for $3-6 \mathrm{~d}$. The yeast cell carrying $\mathrm{AD} / \mathrm{BD}$ vectors were used as a negative control. The yeast cell carrying AD/LhbHLH2 and AD/LhbHLH2260 grew on $\mathrm{SD} /$-Trp/-Leu/-His/-Ade suggesting that the full-length amino acids of LhbHLH2 and the C-terminal 260 amino acids fragment of LhbHLH2 were self-activated. The yeast cell carrying AD/LhbHLH2-420 and LhWDR/ $\mathrm{BD}$ could not grow on $\mathrm{SD} /$-Trp/-Leu/-His/-Ade, suggesting that the N-terminal 420 amino acids fragment of LhbHLH2 and the full-length amino acids of LhWDR were not self-activated. The yeast cell carrying LhWDR/ LhbHLH2 420 grew on SD/-Trp/-Leu/-His/-Ade and the 5-Bromo-4-chloro-3-indolyl $\beta$-Dgalactopyranoside (X-Gal) was blue, indicating that the full-length LhWDR could interact with the N-terminal 420 amino acids of the LhbHLH2 protein. $\mathrm{AD}=$ pGADT7 vector, $\mathrm{BD}=$ pGBKT7 vector.

which, TTG1 is essential for the accumulation of purple anthocyanins in leaves and stems, as well as the development of hairs and root hairs (Walker et al., 1999). The fragment of LhWD40a gene obtained by Suzuki et al. (2016) through analyzing RNA-seq also belongs to the PAC1 clade. Overexpression of homologous genes from the PAC1 cluster proteins, such as VvWDR1, GhTTG1/3, and Setaria italica TTG1, in A. thaliana ttg1 mutant plants has been found to restore many of the mutant phenotypes (Humphries et al., 2005; Liu et al., 2017; Matus et al., 2010). In contrast, overexpression of the genes VvWDR2, G. hirsutum TRANSPARENT TESTA GLABRA 2 (GhTTG2), and GhTTG4, which are clustered in the MP1 clade, was found to be ineffective in restoring $\operatorname{tg} 1$ phenotypes (Humphries et al., 2005; Matus et al., 2010). Although LhWDR, which is also an MP1 clade member, has been observed to increase the expression level of $D F R$ when ectopically expressed in Arabidopsis, LhWDR cannot complement anthocyanin and trichome defects in the ttgl mutant (data not shown). These results thus indicate that, although both MP1 and PAC1 contain WD40 family proteins related to anthocyanin synthesis, the two clades are not functionally redundant. The fragment of $L h W D 40 a$ gene obtained by Suzuki et al. (2016) through analyzing RNA-seq also belongs to the PAC1 clade.

REgulation OF LHWDR in anTHOCYANIN SYNTHESIS. Anthocyanin biosynthesis is generally regulated by the MBW complex, and the ratios and amounts of $R 2 R 3-M Y B, b H L H$, and $W D R$ transcripts determine the amount of anthocyanin produced (De Majnik et al., 1998). In Asiatic hybrid lilies, changes in the expression levels of LhMYB 12 have been found to be consistent with changes in the anthocyanin contents in tepals, filaments, and styles (Yamagishi et al., 2010). Moreover, the transcription of $L h b H L H 2$ has been detected in most tissues and found to be positively correlated with anthocyanin accumulation (Nakatsuka et al., 2009). In the present study, we observed that $L h W D R$ is transcribed in most organs of 'Sorbonne', and high transcript levels were observed in bulb scales and tepals, which are the two pigmented organs in 'Sorbonne'. Anthocyanins are the main visible pigments in Oriental hybrid lily flowers (Yamagishi, 2011), and the observed correlation between the expression levels of $L h W D R$ and pigmentation suggest that $L h W D R$ might be associated with anthocyanin synthesis. In lilies, LhCHS, LhCHI, LhDFR, and LhANS are the major enzymes involved in the synthesis of anthocyanin pigments, with the highest expression levels of these genes being detected in the S4 or S5 tepals. LhMYB 12 has been found to directly activate the LhCHSa and LhDFR genes (Lai et al., 2012; Yamagishi, 2011), and LhDFR transcription has been demonstrated to be associated with the activity of LhbHLH2 (Nakatsuka et al., 2009). In the present study, we found that LhWDR, LhMYB12, LhbHLH2, LhDFR, and LhANS showed similar patterns of expression, which might indicate interactions or regulatory relationships among these genes. When we overexpressed $L h W D R$ in A. thaliana, although we detected no epidermal or anthocyanin-related abnormalities in the transgenic plants, the expression level of the $D F R$ gene increased with overexpression of $L h W D R$, and the expression of $G L 3$ was also slightly upregulated. $D F R$ is a late gene of flavanol biosyntheses and is necessary for anthocyanin biosynthesis, and $A N S$ is located downstream of DFR and directly controls the synthesis of anthocyanins. The expression of $A N S$ is directly related to the amounts of anthocyanins, and in the present study, we found that whereas the overexpression of $L h W D R$ in $A$. thaliana increased the expression of the $D F R$ gene, it did not significantly affect the expression of $A N S$, nor did it alter the content of anthocyanin. We accordingly suspect that $L h W D R$ might participate in anthocyanin biosynthesis pathways by regulating the $D F R$ gene.

INTERACTION BETWEEN LHWDR AND LHBHLH2. In Arabidopsis, both GL3 and EGL3 encode bHLH proteins, which interact with TTG1 (Zhang et al., 2003). The gl3 egl3 double mutant has been found to exhibit phenotypes similar to those of the $\operatorname{tg} 1$ mutant, such as defects in anthocyanin production, seedcoat mucilage production, and root hair growth (Zhang et al., 2003). MYB proteins in A. thaliana, such as GL1, PAP1, PAP 2, CAPRICE, and TRIPTYCHON, form heterodimers with GL3, and in the MBW model, which is dependent on TTG1, there is more than one member of the MYB and bHLH protein families involved and functional redundancy within the same family (Zhang et al., 2003). Although both LhbHLH1 and LhbHLH2 are bHLH-type TFs in lilies, only LhbHLH2 is involved in mediating anthocyanin metabolism (Nakatsuka et al., 2009). Similarly, LhMYB6 and LhMYB12 
are MYB proteins that mediate anthocyanin metabolism in lilies, and $\mathrm{Y} 2 \mathrm{H}$ analysis has revealed that both these proteins interact with LhbHLH2 (Yamagishi et al., 2010).

In the present study, we used the $\mathrm{Y} 2 \mathrm{H}$ assay to examine the relationship between $L h W D R$ and $L h b H L H 2$. We accordingly found that that the C-terminal 240 amino acids of LhbHLH2 exhibited self-activation, and therefore used the remaining 420amino acid sequence as bait, which was observed to interact with the full-length LhWDR. In addition, we examined the interaction between the WD repeat and MYB TFs and found that LhWDR interacted with neither LhMYB6 nor LhMYB12 in the yeast system (data not shown). TFs and coactivators must enter the nucleus to exert regulatory control (Matus et al., 2010), and LhbHLH2 has been shown to be nuclear-localized (Li et al., 2014; Nakatsuka et al., 2009). In contrast, we found that LhWDR is localized in the cytoplasm. Thus, LhWDR would need to enter the nucleus to interact with LhbHLH2 and regulate the expression of downstream genes. This process should be further investigated. Anthocyanin biosynthesis in different tissues is considered to be determined by combinations of the R2R3-MYB, $b H L H$, and $W D R$ factors and the interactions of these factors, which control the expression of a set of structural genes (Morita et al., 2006). Further examination of the MBW complex will be valuable for elucidating the role of this complex in anthocyanin biosynthesis.

\section{Literature Cited}

Albert, N.W., K.M. Davies, D.H. Lewis, H. Zhang, M. Montefiori, C. Brendolise, M.R. Boase, H. Ngo, P.E. Jameson, and K.E. Schwinn. 2014. A conserved network of transcriptional activators and repressors regulates anthocyanin pigmentation in eudicots. Plant Cell 26:962-980.

Albert, N.W., D.H. Lewis, H. Zhang, K.E. Schwinn, P.E. Jameson, and K.M. Davies. 2011. Members of an R2R3-MYB transcription factor family in Petunia are developmentally and environmentally regulated to control complex floral and vegetative pigmentation patterning. Plant J. 65:771-784.

Baudry, A., M.A. Heim, B. Dubreucq, M. Caboche, B. Weisshaar, and L. Lepiniec. 2004. TT2, TT8, and TTG1 synergistically specify the expression of BANYULS and proanthocyanidin biosynthesis in Arabidopsis thaliana. Plant J. 39:366-380.

Bechtold, N. and G. Pelletier. 1998. In planta Agrobacterium mediated transformation of adult Arabidopsis thaliana plants by vacuum infiltration, p. 259-266. In: I. Potrykus and G. Spangenberg (eds.). Gene transfer to plants. Springer, Berlin/Heidelberg, Germany.

Carey, C.C., J.T. Strahle, D.A. Selinger, and V.L. Chandler. 2004. Mutations in the pale aleurone colorl regulatory gene of the Zea mays anthocyanin pathway have distinct phenotypes relative to the functionally similar TRANSPARENT TESTA GLABRA1 gene in Arabidopsis thaliana. Plant Cell 16:450-464.

Causier, B. and B. Davies. 2002. Analysing protein-protein interactions with the yeast two-hybrid system. Plant Mol. Biol. 50:855-870.

De Majnik, J., G. Tanner, R. Joseph, P. Larkin, J. Weinman, M. Djordjevic, and B. Rolfe. 1998. Transient expression of maize anthocyanin regulatory genes influences anthocyanin production in white clover and peas. Funct. Plant Biol. 25:335-343.

de Vetten, N., F. Quattrocchio, J. Mol, and R. Koes. 1997. The an11 locus controlling flower pigmentation in petunia encodes a novel WD-repeat protein conserved in yeast, plants, and animals. Genes Dev. 11:1422-1434.

del March Naval, M., F. Gil-Muñoz, A. Lloret, C. Besada, A. Salvador, M.L. Badenes, and G. Ríos. 2016. A WD40-repeat protein from persimmon interacts with the regulators of proanthocyanidin biosynthesis DkMYB2 and DkMYB4. Tree Genet. Genomes 12:1-11.

Fatihah, H.N., D.M. López, G. Van Arkel, J.G. Schaart, R. Visser, and F.A. Krens. 2019. The ROSEA1 and DELILA transcription factors control anthocyanin biosynthesis in Nicotiana benthamiana and Lilium flowers. Scientia Hort. 243:327-337.

Feller, A., K. Machemer, E.L. Braun, and E. Grotewold. 2011. Evolutionary and comparative analysis of MYB and bHLH plant transcription factors. Plant J. 66:94-116.

Gonzalez, A., M. Zhao, J.M. Leavitt, and A.M. Lloyd. 2008. Regulation of the anthocyanin biosynthetic pathway by the TTG1/bHLH/Myb transcriptional complex in Arabidopsis seedlings. Plant J. 53:814-827.

Gould, K., J. McKelvie, and K. Markham. 2002. Do anthocyanins function as antioxidants in leaves? Imaging of $\mathrm{H}_{2} \mathrm{O}_{2}$ in red and green leaves after mechanical injury. Plant Cell Environ. 25:1261-1269.

Grotewold, E. 2006. The genetics and biochemistry of floral pigments. Annu. Rev. Plant Biol. 57:761-780.

Humphries, J.A., A.R. Walker, J.N. Timmis, and S.J. Orford. 2005. Two WD-repeat genes from cotton are functional homologues of the Arabidopsis thaliana TRANSPARENT TESTA GLABRA1 (TTG1) gene. Plant Mol. Biol. 57:67-81.

Kamran, M.A., R. Mufti, N. Mubariz, J.H. Syed, A. Bano, M.T. Javed, M.F.H. Munis, Z. Tan, and H.J. Chaudhary. 2014. The potential of the flora from different regions of Pakistan in phytoremediation: A review. Environ. Sci. Pollut. Res. 21:801-812.

Lai, Y.S., H.X. Li, and M. Yamagishi. 2013. A review of target gene specificity of flavonoid R2R3-MYB transcription factors and a discussion of factors contributing to the target gene selectivity. Front. Biol. 8:577-598.

Lai, Y.S., Y. Shimoyamada, M. Nakayama, and M. Yamagishi. 2012. Pigment accumulation and transcription of LhMYB12 and anthocyanin biosynthesis genes during flower development in the Asiatic hybrid lily (Lilium spp.). Plant Sci. 193:136-147.

Li, W., B. Wang, M. Wang, M. Chen, J.M. Yin, G.M. Kaleri, R.J. Zhang, T.N. Zuo, X. You, and Q. Yang. 2014. Cloning and characterization of a potato StAN11 gene involved in anthocyanin biosynthesis regulation. J. Integr. Plant Biol. 56:364-372.

Lim, K.B., R. Barba-Gonzalez, S. Zhou, M.S. Ramanna, and J.M. van Tuyl. 2008. Interspecific hybridization in lily (Lilium): Taxonomic and commercial aspects of using species hybrids in breeding, p. 146151. In: J.A. Teixeira da Silva (ed.). Floriculture, ornamental and plant biotechnology. Global Sci. Books, Ikenobe, Japan.

Liu, K., S. Qi, D. Li, C. Jin, C. Gao, S. Duan, B. Feng, and M. Chen. 2017. TRANSPARENT TESTA GLABRA 1 ubiquitously regulates plant growth and development from Arabidopsis to foxtail millet (Setaria italica). Plant Sci. 254:60-69.

Liu, J.X., R. Srivastava, P. Che, and S.H. Howell. 2007. An endoplasmic reticulum stress response in Arabidopsis is mediated by proteolytic processing and nuclear relocation of a membrane-associated transcription factor, bZIP28. Plant Cell 19:4111-4119.

Lloyd, A., A. Brockman, L. Aguirre, A. Campbell, A. Bean, A. Cantero, and A. Gonzalez. 2017. Advances in the MYB-bHLH-WD repeat (WBW) pigment regulatory model: Addition of a WRKY factor and co-option of an anthocyanin MYB for betalain regulation. Plant Cell Physiol. 58:1431-1441.

Matus, J., M. Poupin, P. Canon, E. Bordeu, J. Alcalde, and P. ArceJohnson. 2010. Isolation of WDR and bHLH genes related to flavonoid synthesis in grapevine (Vitis vinifera L.). Plant Mol. Biol. 72:607-620.

Mol, J., G. Jenkins, E. Schäfer, D. Weiss, and V. Walbot. 1996. Signal perception, transduction, and gene expression involved in anthocyanin biosynthesis. Crit. Rev. Plant Sci. 15:525-557.

Morita, Y., M. Saitoh, A. Hoshino, E. Nitasaka, and S. Iida. 2006. Isolation of cDNAs for R2R3-MYB, bHLH and WDR transcriptional regulators and identification of $c$ and $c a$ mutations conferring white flowers in the japanese morning glory. Plant Cell Physiol. 47:457-470. Nakatsuka, A., M. Yamagishi, M. Nakano, K. Tasaki, and N. Kobayashi. 2009. Light-induced expression of basic helix-loop-helix genes involved in anthocyanin biosynthesis in flowers and leaves of Asiatic hybrid lily. Scientia Hort. 121:84-91.

Neer, E.J., C.J. Schmidt, R. Nambudripad, and T.F. Smith. 1994. The ancient regulatory-protein family of WD-repeat proteins. Nature 371:297-300. 
Nie, X., Y. Zhao, L. Zhang, Y. Tang, J. Wang, and Z. Zhao. 2015. Mechanisms of MYB-bHLH-WD40 complex in the regulation of anthocyanin biosynthesis in plants. Agr. Biotechnol. 4:5.

Rabino, I. and A.L. Mancinelli. 1986. Light, temperature, and anthocyanin production. Plant Physiol. 81:922-924.

Quattrocchio, F., J. Wing, K. van der Woude, E. Souer, N. de Vetten, J. Mol, and R. Koes. 1999. Molecular analysis of the anthocyanin2 gene of petunia and its role in the evolution of flower color. Plant Cell 11:1433-1444.

Saitou, N. and M. Nei. 1987. The neighbor-joining method: A new method for reconstructing phylogenetic trees. Mol. Biol. Evol. 4:406-425.

Schwinn, K., J. Venail, Y. Shang, S. Mackay, V. Alm, E. Butelli, R. Oyama, P. Bailey, K. Davies, and C. Martin. 2006. A small family of $M Y B$-regulatory genes controls floral pigmentation intensity and patterning in the genus Antirrhinum. Plant Cell 18:831-851.

Smith, T.F., C. Gaitatzes, K. Saxena, and E.J. Neer. 1999. The WD repeat: A common architecture for diverse functions. Trends Biochem. Sci. 24:181-185.

Sompornpailin, K., Y. Makita, M. Yamazaki, and K. Saito. 2002. A WD-repeat-containing putative regulatory protein in anthocyanin biosynthesis in Perilla frutescens. Plant Mol. Biol. 50:485-495.

Spelt, C., F. Quattrocchio, J.N. Mol, and R. Koes. 2000. Anthocyanin1 of petunia encodes a basic helix-loop-helix protein that directly activates transcription of structural anthocyanin genes. Plant Cell 12:1619-1631.

Suzuki, K., T. Suzuki, T. Nakatsuka, H. Dohra, M. Yamagishi, K. Matsuyama, and H. Matsuura. 2016. RNA-seq-based evaluation of bicolor tepal pigmentation in Asiatic hybrid lilies (Lilium spp.). BMC Genomics 17:611.

van Nocker, S. and P. Ludwig. 2003. The WD-repeat protein superfamily in Arabidopsis: Conservation and divergence in structure and function. BMC Genomics 4:50.

Walker, A.R., P.A. Davison, A.C. Bolognesi-Winfield, C.M. James, N. Srinivasan, T.L. Blundell, J.J. Esch, M.D. Marks, and J.C. Gray. 1999. The TRANSPARENT TESTA GLABRA1 locus, which regulates trichome differentiation and anthocyanin biosynthesis in Arabidopsis, encodes a WD40 repeat protein. Plant Cell 11:1337-1349.

Wang, X. and M. Yamagishi. 2019. Mechanisms suppressing carotenoid accumulation in flowers differ depending on the hybrid groups of lilies (Lilium spp.). Scientia Hort. 243:159-168.

Winkel-Shirley, B. 2001. Flavonoid biosynthesis. A colorful model for genetics, biochemistry, cell biology, and biotechnology. Plant Physiol. 126:485-493.

Xu, W.J., C. Dubos, and L. Lepiniec. 2015. Transcriptional control of flavonoid biosynthesis by MYB-bHLH-WDR complexes. Trends Plant Sci. 20:176-185.

Yamagishi, M. 2011. Oriental hybrid lily Sorbonne homologue of LhMYB12 regulates anthocyanin biosyntheses in flower tepals and tepal spots. Mol. Breed. 28:381-389.
Yamagishi, M. 2018. Involvement of a LhMYB18 transcription factor in large anthocyanin spot formation on the flower tepals of the Asiatic hybrid lily (Lilium spp.) cultivar "Grand Cru". Mol. Breeding. 38:60.

Yamagishi, M. and K. Akagi. 2013. Morphology and heredity of tepal spots in Asiatic and Oriental hybrid lilies (Lilium spp.). Euphytica 194:325-334.

Yamagishi, M. 2016. A novel R2R3-MYB transcription factor regulates light-mediated floral and vegetative anthocyanin pigmentation patterns in Lilium regale. Mol. Breed. 36:1-14.

Yamagishi, M., H. Ihara, K. Arakawa, S. Toda, and K. Suzuki. 2014a. The origin of the LhMYB12 gene, which regulates anthocyanin pigmentation of tepals, in Oriental and Asiatic hybrid lilies (Lilium spp.). Scientia Hort. 174:119-125.

Yamagishi, M., Y. Shimoyamada, T. Nakatsuka, and K. Masuda. 2010. Two R2R3-MYB genes, homologs of petunia AN2, regulate anthocyanin biosyntheses in flower tepals, tepal spots and leaves of Asiatic hybrid lily. Plant Cell Physiol. 51:463-474.

Yamagishi, M., S. Toda, and K. Tasaki. 2014b. The novel allele of the LhMYB12 gene is involved in splatter-type spot formation on the flower tepals of Asiatic hybrid lilies (Lilium spp.). New Phytol. 201:1009-1020.

Yamagishi, M., H. Uchiyama, and T. Handa. 2018. Floral pigmentation pattern in Oriental hybrid lily (Lilium spp.) cultivar 'Dizzy' is caused by transcriptional regulation of anthocyanin biosynthesis genes. J. Plant Physiol. 228:85-91.

Yamagishi, M., Y. Yoshida, and M. Nakayama. 2012. The transcription factor LhMYB12 determines anthocyanin pigmentation in the tepals of Asiatic hybrid lilies (Lilium spp.) and regulates pigment quantity. Mol. Breed. 30:913-925.

Yang, K.Z., C. Xia, X.L. Liu, X.Y. Dou, W. Wang, L.Q. Chen, X.Q. Zhang, L.F. Xie, L. He, and X. Ma. 2009. A mutation in Thermosensitive Male Sterile 1, encoding a heat shock protein with DnaJ and PDI domains, leads to thermosensitive gametophytic male sterility in Arabidopsis. Plant J. 57:870-882.

Yang, P. and W.S. Sale. 1998. The Mr 140,000 intermediate chain of Chlamydomonas flagellar inner arm dynein is a WD-repeat protein implicated in dynein arm anchoring. Mol. Biol. Cell 9:3335-3349.

Yao, P., H. Zhao, X. Luo, F. Gao, H. Li, H. Yao, H. Chen, S. Park, and Q. Wu. 2017. Fagopyrum tataricum FtWD40 functions as a positive regulator of anthocyanin biosynthesis in transgenic tobacco. J. Plant Growth Regul. 36:755-765.

Zhang, F., A. Gonzalez, M. Zhao, C.T. Payne, and A. Lloyd. 2003. A network of redundant bHLH proteins functions in all TTG1-dependent pathways of Arabidopsis. Development 130:4859-4869. 